\title{
Overall Control Strategy of Grid Connected to Wind Farm Using FACTS
}

\author{
Qusay. Salem
}

\begin{abstract}
Wind energy is attracting more attention from researchers and even utilities due to its benefits as a clean and abundant source of energy. In this paper a comprehensive and intensive study of a wind farm connected to the grid has been proposed. The aim of this paper is to investigate the system stability of a wind farm connected to medium voltage grid in different operating conditions and to investigate the behavior of the system when using a Static Var Compensator (SVC) and Static Synchronous Compensator (STATCOM). A wind farm of $12 \mathrm{MW}$ consisting of four wind turbines connected to medium voltage grid has been proposed. Wind turbines, transmission systems, transformers and grid models as well as SVC and STATCOM models are all developed in SimPowerSystems library in MATLAB / SIMULINK. It was observed from the simulation results that STATCOM and SVC support the system voltage and reactive power in case of healthy conditions. However, it was noticed that they considerably improve the system stability during and especially after severe disturbances.
\end{abstract}

Keywords-Wind Power Generation, Medium Voltage Grid, FACTS Devices, System Control

\section{INTRODUCTION}

$\mathrm{W}$ ind energy has confirmed to be a copious, clean, and purely renewable source of power. It is frugal to use in producing power in rural areas [1]. In sustainable energy system, the key paradigm is attained by energy conservation and the utilization of renewable source. The appeal to consolidate the renewable energy such as wind energy into power system is to make it possible to depress the environmental effect on conventional effect [2]. Technical challenges and consideration of stability, voltage regulation power quality problems are presented in case of integration of wind energy into existing power system [3].

Wind energy system converts the obtainable kinetic energy in the wind into mechanical energy that can spur an electrical generator. Predominantly, wind power generators are self excited induction generators. Induction generators characteristics are strongly affected by the massive reactive power absorbed during their normal operating conditions. This reactive power trouble may engender dynamic voltage instability in the system. The needed reactive power of induction generator can be provided either by the grid or self

Qusay. $\quad \begin{gathered}\text { Salem, } \\ \text { qusi_salim@yahoo.com }\end{gathered}$
Yarmouk University, Jordan.

DOI: 10.9756/BIJPSIC.10289 capacitor bank in parallel with the generator stator terminals. Dynamic reactive power compensators are always required to stabilize the voltage and to supply the desired reactive power at wind generator bus under normal and abnormal operation. Flexible Alternating Current Transmission Systems (FACTS) can be very profitable to simultaneously deliver reactive power and support bus voltage at wind generator interface [4]. In the literature, there are several research papers that support such kind of study. A wind farm modeling with integration of STATCOM is studied in [5]. Investigation and analysis of grid connected to fixed speed wind power generation system is also studied in [6]. In [7], it was investigated that system instability of wind farms based on fixed speed induction generators is largely caused by the excessive reactive power absorption by the fixed speed induction generators after fault due to the large rotor slip gained during fault. It was found that the SVC and STATCOM considerably improve the system stability during and after disturbances. In [8], transient stability of a wind farm based on conventional fixed speed induction generator when it has been integrated with a weak grid has been studied.

This research paper has studied a normal operation case and a faulty case (two successive faults) occurring on the transmission line at point of common coupling. The impact of integration of STATCOM and SVC in improving the transient stability of the wind farm during the faulty conditions is considered. A performance comparison between the two kinds of FACTS is also considered.

\section{Modeling AND Structure OF THE Wind TURBINE}

Wind turbine blades are turned as the wind strikes, and then the generator rotor turns to generate electricity. Wind speed and rotor size are the main parameters that the output power of the wind turbine is related. This power is proportional to the cubic wind speed, assuming all other parameters are constant. Thus, as the wind speed increases the output power of wind turbines will increase significantly [9] [10].

The block diagram of the wind turbine induction generator (WTIG) is shown in Fig. 1. The stator winding is connected directly to the $60 \mathrm{HZ}$ grid and the rotor is driven by a variable pitch wind turbine. The power captured by the wind turbine is converted into electrical power by the induction generator and is transmitted to the grid by the stator winding. The pitch angle is controlled in order to limit the generator output power to its nominal value for high wind speeds. In order to generate power the induction generator speed must be slightly above the synchronous speed. Speed varies approximately between $1 \mathrm{pu}$ at no load and $1.005 \mathrm{pu}$ at full load. The pitch angle controller regulates the wind turbine blade pitch angle $\beta$, according to the wind speed variations. 
Hence, the power output of WTIG depends on the characteristics of the pitch controller in addition to the turbine and generator characteristics. This control guarantees that, irrespective of the voltage, the power output of the WTIG for any wind speed will be equal to the designed value for that speed. This designed power output of the WTIG with wind speed is provided by the manufacturer in the form of a power curve. Hence, for a given wind speed, power output can be obtained from the power curve of the WTIG. The pitch angle $\beta$ is controlled in order to limit the generator output power at its nominal value for winds exceeding the nominal speed. $\beta$ is controlled by a Proportional-Integral (PI) controller in order to limit the electric output power to the nominal mechanical power. When the measured electric output power is under its nominal value, $\beta$ is kept constant at zero degree. When it increases above its nominal value the PI controller increases $\beta$ to bring back the measured power to its nominal value. The pitch angle control system is shown in Fig. 2. [11].

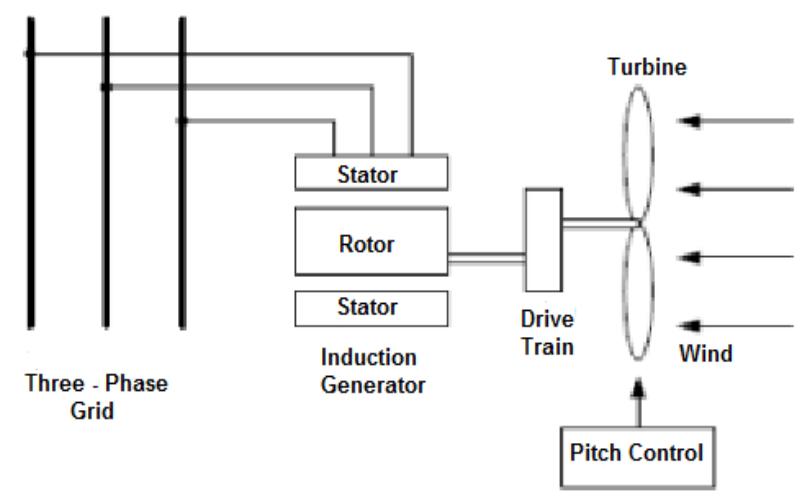

Figure 1: Block Diagram of Wind Turbine Induction Generator (WTIG)

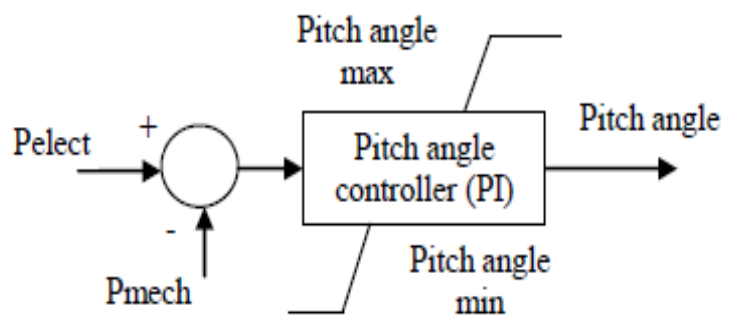

Figure 2: Pitch angle control system

\section{MOdELING AND STRUCTURE OF STATCOM AND SVC}

The Static Synchronous Compensator (STATCOM) and Static Var Compensator (SVC) are shunt devices of the Flexible AC Transmission Systems (FACTS) family. These devices use power electronics to control power flow and improve transient stability on power grids. Providing dynamic reactive power compensation using SVC and STATCOM can possibly raise the network voltage during and after fault.

The SVC regulates voltage at its terminals by controlling the amount of reactive power injected into or absorbed from the power system. When system voltage is low, the SVC generates reactive power. When system voltage is high, it absorbs reactive power (SVC inductive). The variation of reactive power is performed by switching three-phase capacitor banks and inductor banks connected on the secondary side of a coupling transformer. Each capacitor bank is switched on and off by three thyristor switches (Thyristor Switched Capacitor or TSC). Reactors are either switched onoff (Thyristor Switched Reactor or TSR) or phase-controlled (Thyristor Controlled Reactor or TCR). Fig. 3 shows a singleline diagram of a static var compensator and a simplified block diagram of its control system [12].

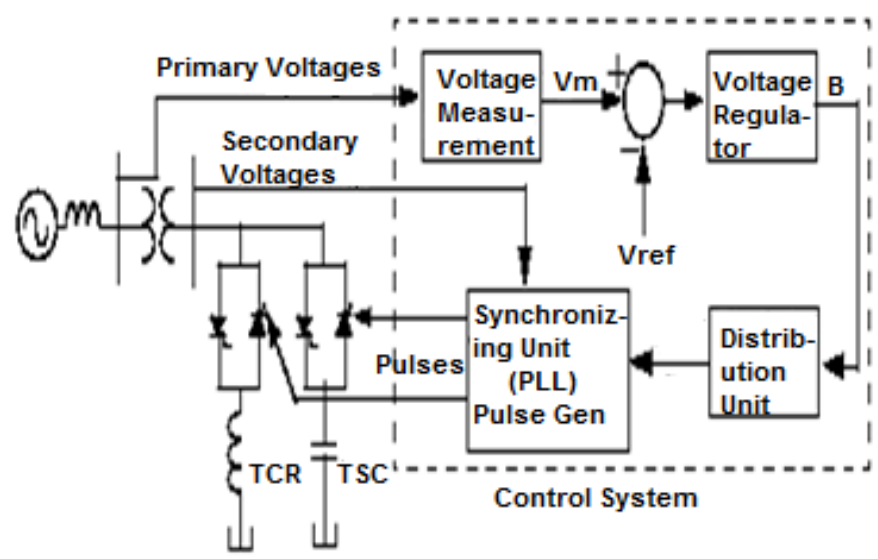

Figure 3: Single line diagram of an SVC and its control system block diagram

The control system consists of four major categories which are a measurement system measuring the positive-sequence voltage to be controlled, a voltage regulator that uses the voltage error (difference between the measured voltage $\mathrm{Vm}$ and the reference voltage $\mathrm{V}$ ref) to determine the SVC susceptance $B$ needed to keep the system voltage constant, a distribution unit that determines the TSCs (and eventually TSRs) that must be switched in and out and computes the firing angle $\alpha$ of TCRs and finally a synchronizing system using a phase-locked loop (PLL) synchronized on the secondary voltages and a pulse generator that send appropriate pulses to the thyristors. The SVC can be operated in two different modes voltage regulation mode (the voltage is regulated within limits as explained below) and var control mode (the SVC susceptance is kept constant). When the SVC is operated in voltage regulation mode, it implements the V-I characteristic shown in Fig. 4. As long as the SVC susceptance $B$ stays within the maximum and minimum susceptance values imposed by the total reactive power of capacitor banks $\left(\mathrm{Bc}_{\max }\right)$ and reactor banks $\left(\mathrm{Bl}_{\max }\right)$, the voltage is regulated at the reference voltage $\mathrm{V}$ ref. However, a voltage droop is normally used (usually between $1 \%$ and $4 \%$ at maximum reactive power output), and the V-I characteristic has the slope indicated in the figure. The V-I characteristic is described by the following three equations [12]. 


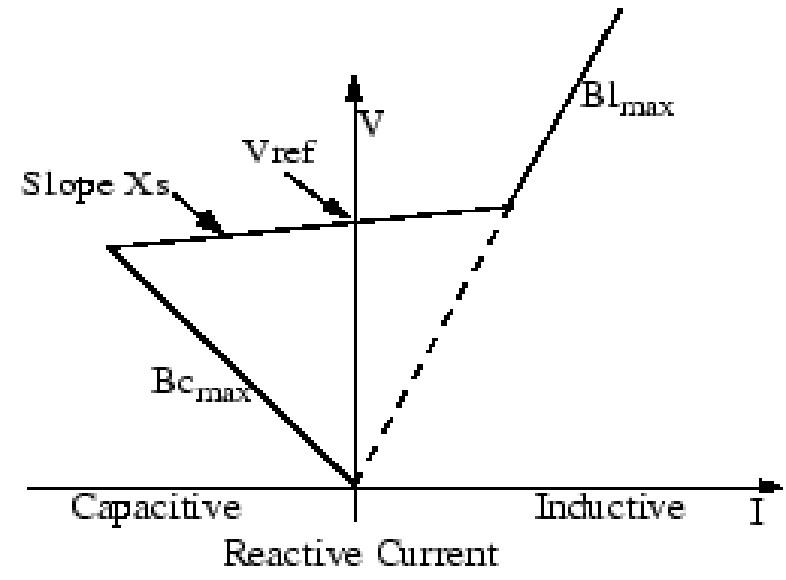

Fig. 4. SVC V-I characteristics

$$
\begin{aligned}
& V=V_{\text {ref }}+X_{S} . I \text { if }\left(-B c_{\max }<B<B I_{\text {max }}\right) \\
& V=-\frac{I}{B c_{\max }} \text { if } B=B c_{\max } \\
& V=\frac{I}{B I_{\max }} \text { if } B=B I_{\max }
\end{aligned}
$$

Where,

$V$ is the positive sequence voltage in pu, $I$ is the reactive current in $\mathrm{pu} / \mathrm{Pbase}, X_{S}$ is the slope or droop reactance in $\mathrm{pu} / \mathrm{Pbase}, B c_{\max }$ is the maximum capacitive susceptance in $\mathrm{pu} / \mathrm{Pbase}, B I_{\max }$ is the maximum inductive susceptance in $\mathrm{pu} / \mathrm{Pbase}$, Pbase is the three phase base power.

Similar to SVC, the STATCOM regulates voltage at its terminal by controlling the amount of reactive power injected into or absorbed from the power system. When system voltage is low, the STATCOM generates reactive power (STATCOM capacitive). When system voltage is high, it absorbs reactive power (STATCOM inductive). The variation of reactive power is performed by means of a Voltage-Sourced Converter (VSC) connected on the secondary side of a coupling transformer. The VSC uses forced-commutated power electronic devices (GTOs, IGBTs or IGCTs) to synthesize a voltage $\mathrm{V} 2$ from a DC voltage source. The principle of operation of the STATCOM is explained in Fig. 5 which shows the active and reactive power transfer between a source $\mathrm{V}_{1}$ and a source $\mathrm{V}_{2}$. In this figure, $\mathrm{V}_{1}$ represents the system voltage to be controlled and $\mathrm{V}_{2}$ is the voltage generated by the VSC. In steady state operation, the voltage V2 generated by the VSC is in phase with $\mathrm{V}_{1}(\delta=0)$, so that only reactive power is flowing $(\mathrm{P}=0)$. If $\mathrm{V}_{2}$ is lower than $\mathrm{V}_{1}, \mathrm{Q}$ is flowing from $\mathrm{V}_{1}$ to $\mathrm{V} 2$ (STATCOM is absorbing reactive power). On the reverse, if $\mathrm{V}_{2}$ is higher than $\mathrm{V}_{1}, \mathrm{Q}$ is flowing from $\mathrm{V}_{2}$ to $\mathrm{V}_{1}$ (STATCOM is generating reactive power) [12]. The amount of reactive power is given by

$$
Q=(V 1(V 1-V 2)) / X
$$

Where,

$\mathrm{V} 1$ is line to line voltage of source $1, \mathrm{~V}_{2}$ is line to line voltage of source $2, X$ is the reactance of interconnection transformer and filters.
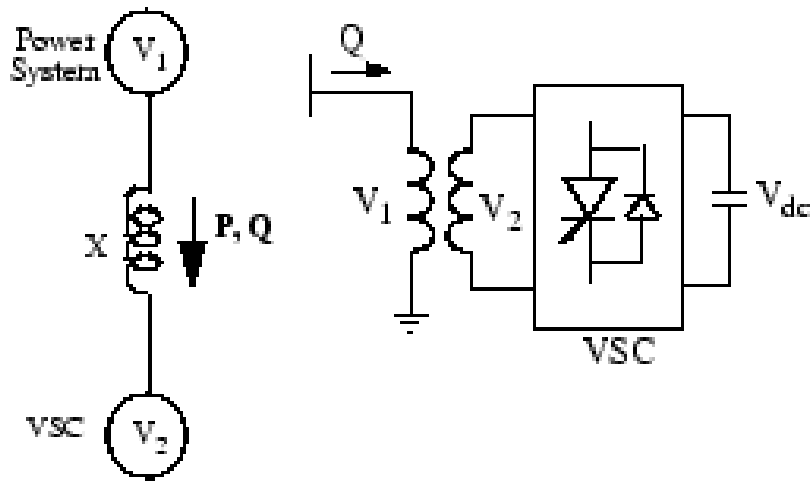

Figure 5: Operating principle of the STATCOM

A capacitor connected on the DC side of the VSC acts as a DC voltage source. In steady state the voltage $V_{2}$ has to be phase shifted slightly behind $\mathrm{V}_{1}$ in order to compensate for transformer and VSC losses and to keep the capacitor charged. Fig. 6 shows a single-line diagram of the STATCOM and a simplified block diagram of its control system. The control system consists of a phase-locked loop (PLL) which synchronizes on the positive-sequence component of the threephase primary voltage $\mathrm{V} 1$. The output of the PLL (angle $\theta=$ $\omega t$ ) is used to compute the direct-axis and quadrature-axis components of the AC three-phase voltage and currents (labeled as $V_{d}, V_{q}$ or $I_{d}, I_{q}$ on the diagram). Measurement systems measuring the $\mathrm{d}$ and $\mathrm{q}$ components of AC positivesequence voltage and currents to be controlled as well as the DC voltage $V_{d c}$ are also used. In addition, an outer regulation loop consisting of an $\mathrm{AC}$ voltage regulator and a $\mathrm{DC}$ voltage regulator is integrated in the control system. The output of the AC voltage regulator is the reference current $I_{\text {qref }}$ for the current regulator $(I q=$ current in quadrature with voltage which controls reactive power flow). The output of the DC voltage regulator is the reference current $I_{\text {dref }}$ for the current regulator $(I d=$ current in phase with voltage which controls active power flow). Finally, an inner current regulation loop consisting of a current regulator would be the last component of the control system. The current regulator controls the magnitude and phase of the voltage generated by the PWM converter $\left(V_{2 d} V_{2 q}\right)$ from the $I_{d r e f}$ and $I_{\text {qref }}$ reference currents produced respectively by the $\mathrm{DC}$ voltage regulator and the $\mathrm{AC}$ voltage regulator (in voltage control mode). The current regulator is assisted by a feed forward type regulator which predicts the $\mathrm{V}_{2}$ voltage output $\left(\begin{array}{lll}V_{2} d & V 2 q\end{array}\right)$ from the $\mathrm{V}_{1}$ measurement $(V 1 d V 1 q)$ and the transformer leakage reactance [12].

The STATCOM can be operated in two different modes which are voltage regulation mode and var control mode. When the STATCOM is operated in voltage regulation mode, it implements the following V-I characteristic shown in Fig. 7. As long as the reactive current stays within the minimum and minimum current values (-Imax, Imax) imposed by the 


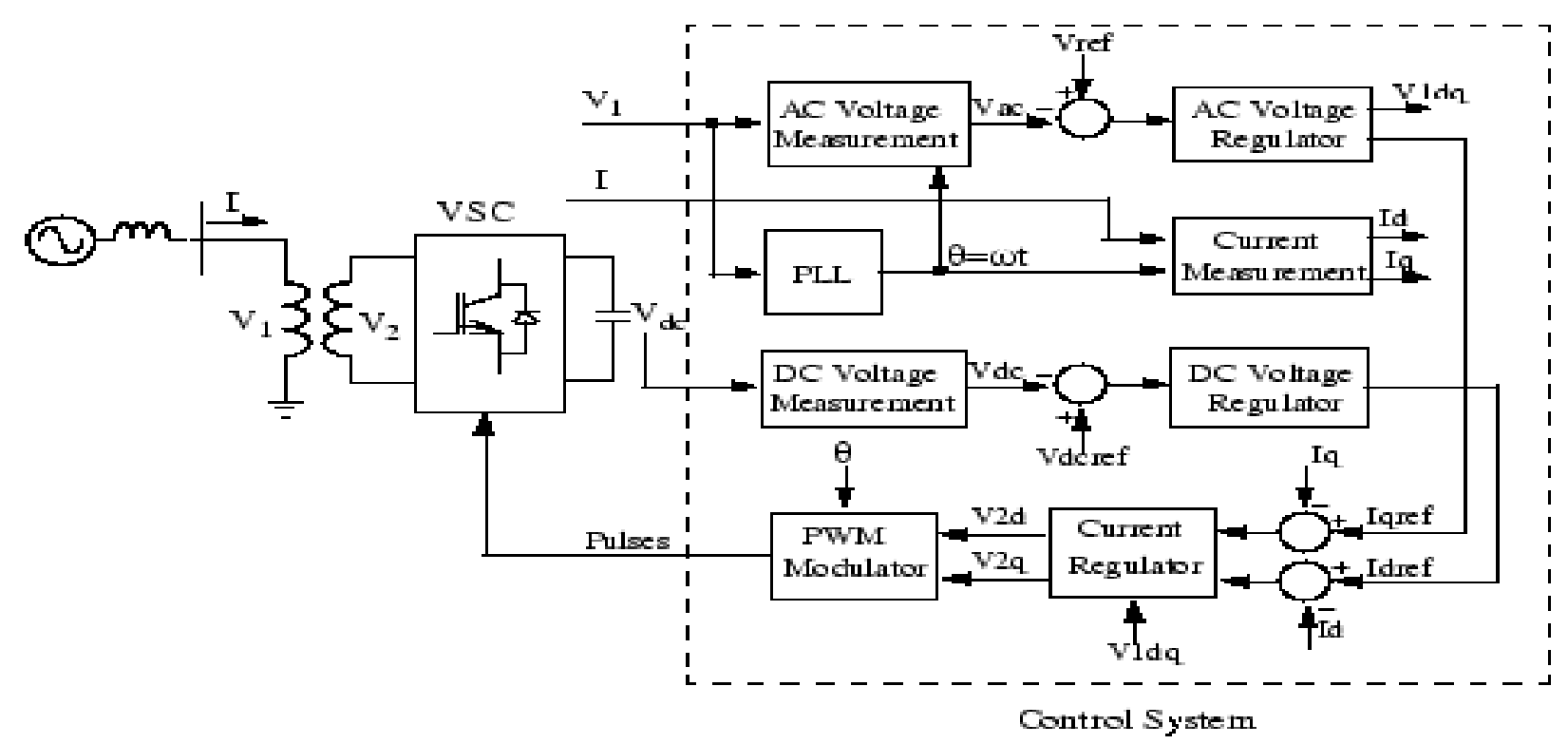

Figure 6: Single line diagram of STATCOM and its control system

converter rating, the voltage is regulated at the reference voltage Vref. However, a voltage droop is normally used (usually between $1 \%$ and $4 \%$ at maximum reactive power output), and the V-I characteristic has the slope indicated in the figure. In the voltage regulation mode, the V-I characteristic is described by the following equation:

$$
V=V_{\text {ref }}+X_{s} \cdot I
$$

Where, $V$ is the positive sequence voltage, $I$ is the reactive current in pu/Pnom, $X_{S}$ is the slope or droop reactance in $\mathrm{pu} / \mathrm{Pnom}$, Pnom is the three phase nominal power of the converter. The STATCOM performs the same function as the SVC. However at voltages lower than the normal voltage regulation range, the STATCOM can generate more reactive power than the SVC. This is due to the fact that the maximum capacitive power generated by a SVC is proportional to the square of the system voltage (constant susceptance) while the maximum capacitive power generated by a STATCOM decreases linearly with voltage (constant current). This ability to provide more capacitive reactive power during a fault is one important advantage of the STATCOM over the SVC. In addition, the STATCOM will normally exhibit a faster response than the SVC because with the VSC, the STATCOM has no delay associated with the thyristor firing (in the order of $4 \mathrm{~ms}$ for a SVC).

\section{SYSTEM STABILITY CONCERNS}

Large wind farms are at most constructed far away from load centers and connected into relatively weak transmission networks. Wind farms presence in such weak transmission networks sustains serious concerns about system security and stability. Wind power integration forced utilities concerns to focus on stability problems rather than power quality issues. The voltage stability as well as the transient stability issues are the main problems in the grid impact studies of wind power integration, because a large proportion of existing wind farms are based on fixed - speed wind turbines (FSWTs) equipped with simple induction generators [13]. As the penetration of wind energy increases, the significance of wind power generation by wind farms is also increase. Production capability will be lost if a large power wind farm is suddenly disconnected. The system may suffer a drop in voltage or frequency and possibly followed by a blackout unless the remaining power plants replace the loss within very short time. As a result, to avoid total disconnection from the grid, there might be a new generation of wind turbines that can ride through the disturbances and faults. It is important to ensure that the wind turbine can restore the normal operation in a simple way and within suitable time in order to keep system stability [14] [15].

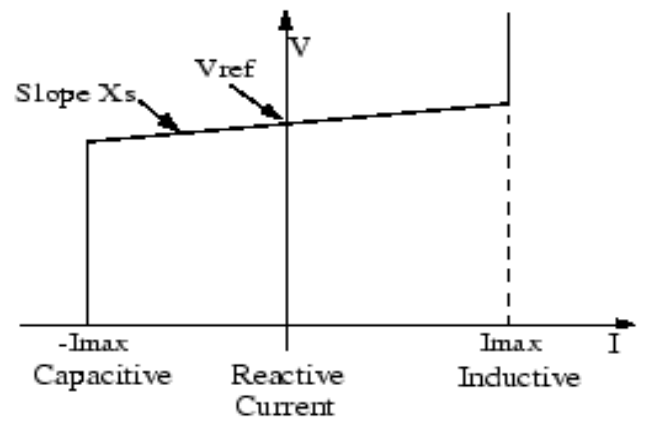

Figure 7: STATCOM V-I characteristics

\section{Test System AND Simulation Results}

Fig. 8 presents the schematic diagram of the proposed system under work. The system consists of a $12 \mathrm{MW}$ wind farm consisting of 4 wind turbines rated at $3 \mathrm{MW}$ for each WTIG; the induction generators connected with the turbines operate at 0.9 power factor. A $25 \mathrm{~km}$ long of overhead transmission lines is considered, a 132/33 KV (47 MVA) 
transformer compromises the grid side, in addition to 4 sets of 33/0.575 KV (4 MVA) transformers for wind farm side. Each WTIG has its own MW rating, 33/0.575 KV transformer, $\mathrm{KVAR}$ rating. The grid is formed by a three - phase balanced A.C voltage source, 2500 MVA short circuit power and (X/R) ratio of 3 at $132 \mathrm{KV}$ voltage. The parameters of all components are presented in tables I, II, III. A wind farm consisting of four wind turbines connected to medium voltage grid is considered firstly without taking into account the occurrence of any short circuit. The generated power is then transferred to the high voltage grid with rated voltage of $132 \mathrm{KV}$ through a $25 \mathrm{Km}$ overhead line. The stator winding of the SCIG is directly connected to the $60 \mathrm{~Hz}$ grid and the rotor is driven by a variable pitch wind turbine. The induction generator speed must be slightly above the synchronous speed so as to generate power. So, speed varies approximately between $1 \mathrm{p} . \mathrm{u}$ at no load and 1.005 p.u at full load. The nominal wind speed producing the nominal mechanical power is $9 \mathrm{~m} / \mathrm{s}$ where 1 p.u equals $3 \mathrm{MW}$.

Table I

Transmission Line Parameters

\begin{tabular}{ccc}
\hline Parameter & Value & Unit \\
\hline Resistance & 0.1153 & $\Omega / k_{m}$ \\
Inductance & 1.05 & $\mathrm{mH} / \mathrm{k}_{m}$ \\
Capacitance & 11.33 & $n \mathrm{~F} / \mathrm{k}_{m}$
\end{tabular}

Table II

Transformer Data

\begin{tabular}{ccc}
\hline Parameter & Value & Unit \\
\hline Wind farm side transformer data $(\mathbf{3 3 / 0 . 5 7 5} \mathbf{~ K V ~})$ \\
Rated power & 4 & $M V A$ \\
$V_{\text {secondary }}-L(R M S)$ & 0.575 & $K_{V}$ \\
$V_{\text {primary }}-L(R M S)$ & 33 & $K_{V}$ \\
Inductance & 0.025 & p.u \\
Grid side transformer data $(\mathbf{1 3 2 / 3 3 ~ K V )}$ & \\
Rated power & 47 & $M V A$ \\
$V_{\text {secondary }} L-L(R M S)$ & 33 & $K_{V}$ \\
$V_{\text {primary }} L-L(R M S)$ & 132 & $K_{V}$ \\
Inductance & 0.025 & $\mathrm{p.u}$ \\
\hline
\end{tabular}

Table III

Wind Farm Parameters

\begin{tabular}{cccc}
\hline Wind Turbine & Symbol & Value & Unit \\
\hline Base power & $S_{B}$ & 3 & $M W$ \\
Base wind & & 9 & $\mathrm{~m} / \mathrm{s}$ \\
speed & $V_{B}$ & 1 & \\
Maximum & & & $\mathrm{p.u}$ \\
power at base & $P_{t \max }$ & 1 & \\
wind speed & & & \\
Base rotational & & & \\
speed & $w_{B}$ & 5,25 & \\
Pitch angle & & & \\
controller gain & $K_{P} K_{i}$ & 45 &
\end{tabular}

\section{Generator}

\begin{tabular}{cccc} 
Base power & $S_{B}$ & $3 / 0.9$ & $M W$ \\
Base voltage & $U_{B}$ & 0.575 & $K_{V}$ \\
Stator resistance & $R_{S}$ & 0.004843 & $p . u$ \\
Stator & & & \\
inductance & $L_{S}$ & 0.1248 & $p . u$ \\
Rotor resistance & $R_{r}$ & 0.004377 & $p . u$ \\
$\quad$ Rotor & & & \\
inductance & $L_{r}$ & 0.1791 & $p . u$ \\
Magnetizing & & & \\
reactance & $L_{m}$ & 6.77 & $p . u$ \\
Inertia Constant & $H$ & 5.04 & $S$ \\
\hline
\end{tabular}

Reactive power compensation varies with the variation in wind speed. Therefore, fixed capacitor banks are assumed to be connected at the terminals of each generator pair but they partly compensate the reactive power absorbed by the induction generators. Consequently, STATCOM or SVC as dynamic var compensators is assumed to be connected at point of common coupling. 


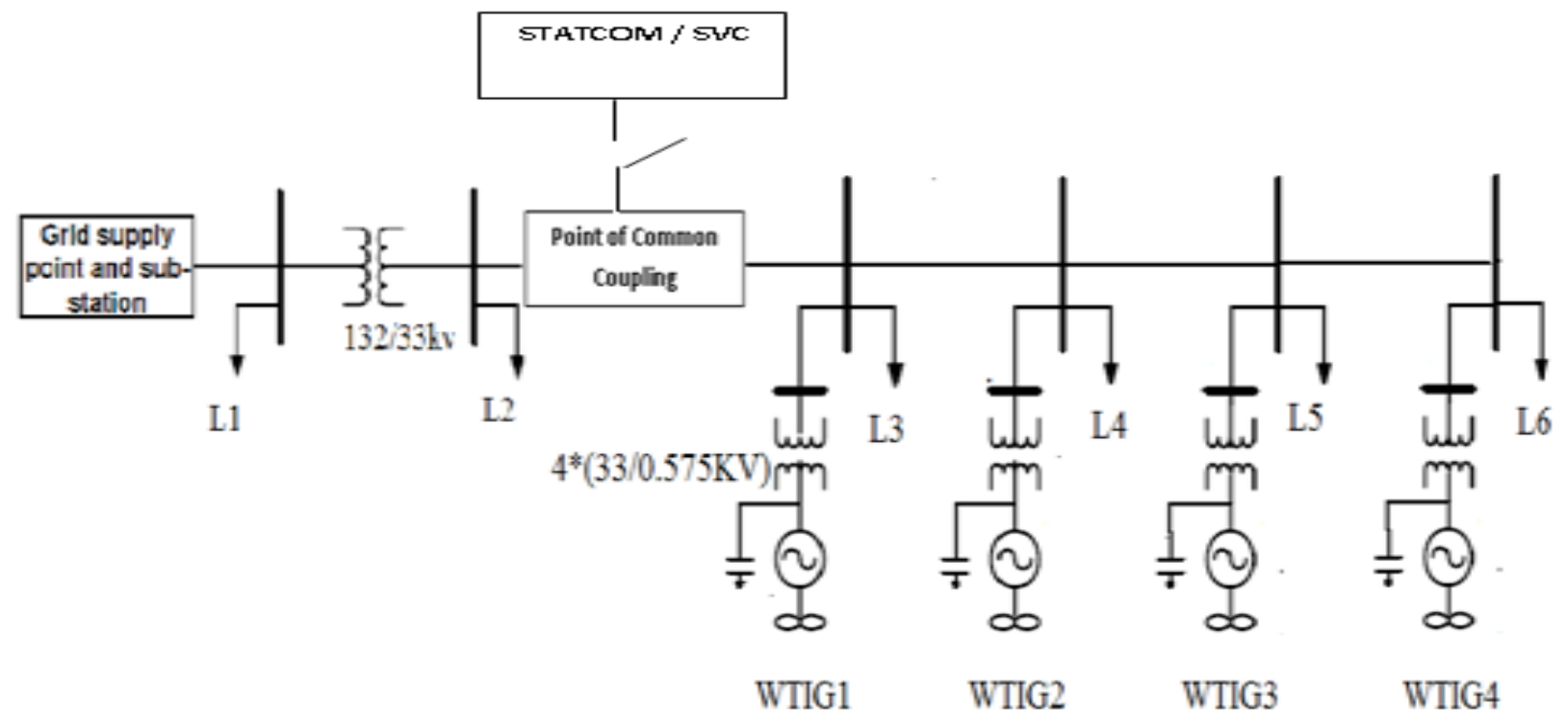

Figure 8: Schematic diagram of the test system

The generated active power, reactive power, voltage at point of common coupling without using FACTS devices (normal case) are shown in Figs 9, 10, 11. As seen from these figures the voltage at point of common coupling approaches 0.954 p.u and the generated active power injected from the wind farm is nearly $6 \mathrm{MW}$ but the reactive power at PCC reaches approximately 4.3 MVAR. In order to support the voltage and provide reactive power compensation at PCC, STATCOOM and SVC should be connected at PCC. Therefore, simulations are carried out with STATCOM and with SVC. It should be noted that the two kinds of FACTS devices in this study give the same performance in healthy conditions.

Fig. 12 shows the simulation results after integrating the STATCOM and SVC to the system. It should be noted that the reactive power at point of common coupling is improved and reaches approximately 1.5 MVAR while it was 4.3 MVAR before adding the FACTS devices. It should also be noted that the two kinds of FACTS devices in this study support the needed reactive power which is absorbed by the induction generators. Both of them give the same performance in improving the reactive power, but it is worth noting that the SVC has less oscillation than STATCOM in reactive power improvement.

Fig. 13 shows also the simulation results after integrating the two kinds of FACTS devices. STATCOM and SVC have approximately the same performance in improving the voltage stability at point of common coupling. The voltage reaches approximately 0.993 p.u while it was 0.954 p.u before adding them to the system. STATCOM and SVC have efficiently supported the system voltage stability. It should be noted that STATCOM has less oscillation than SVC in improving the voltage stability.

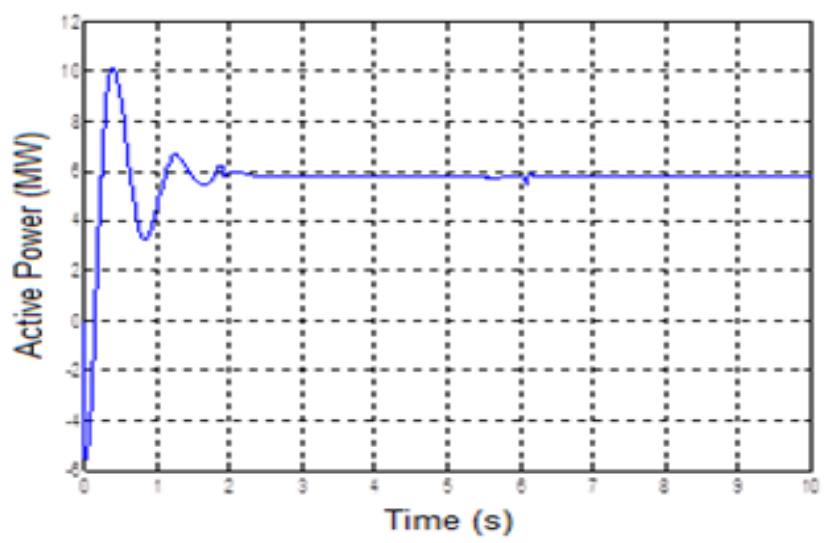

Figure 9: Active Power at PCC

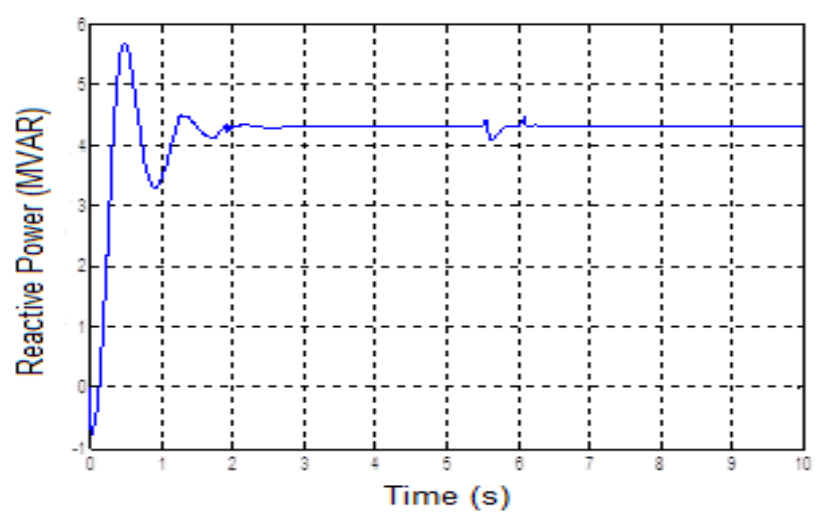

Figure 10: Reactive Power at PCC 


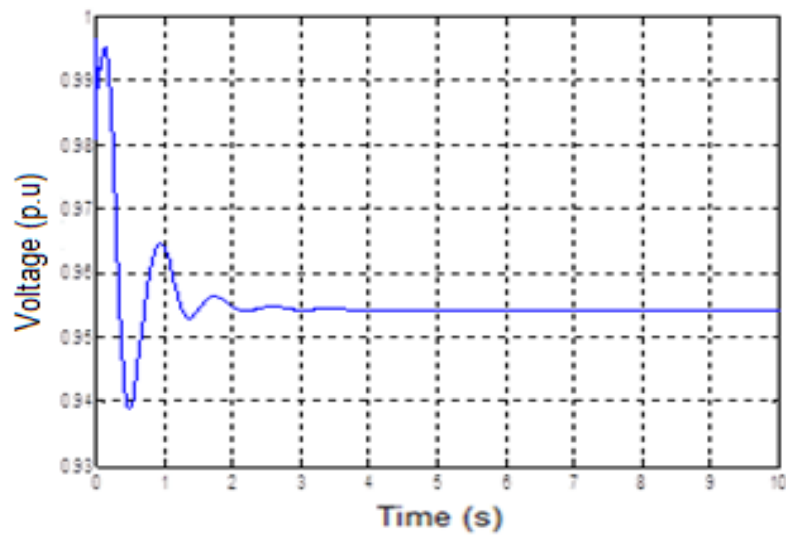

Figure 11: Voltage at PCC

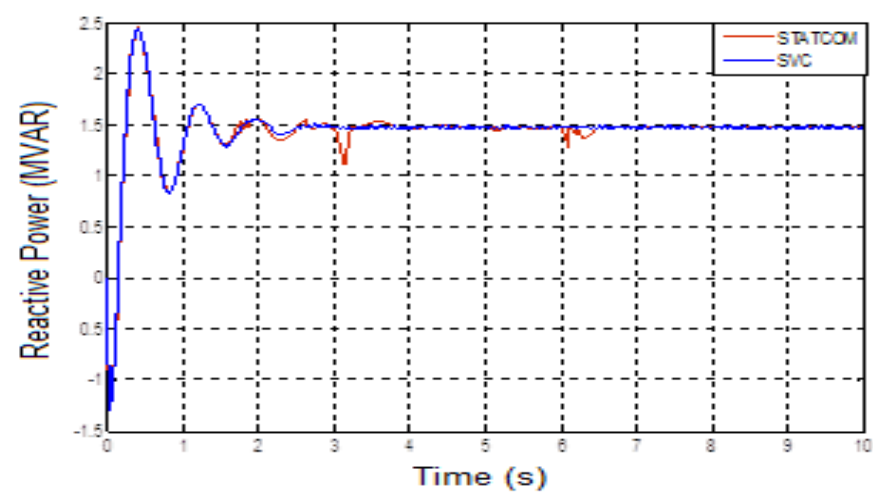

Figure 12: Reactive Power at PCC with FACTs

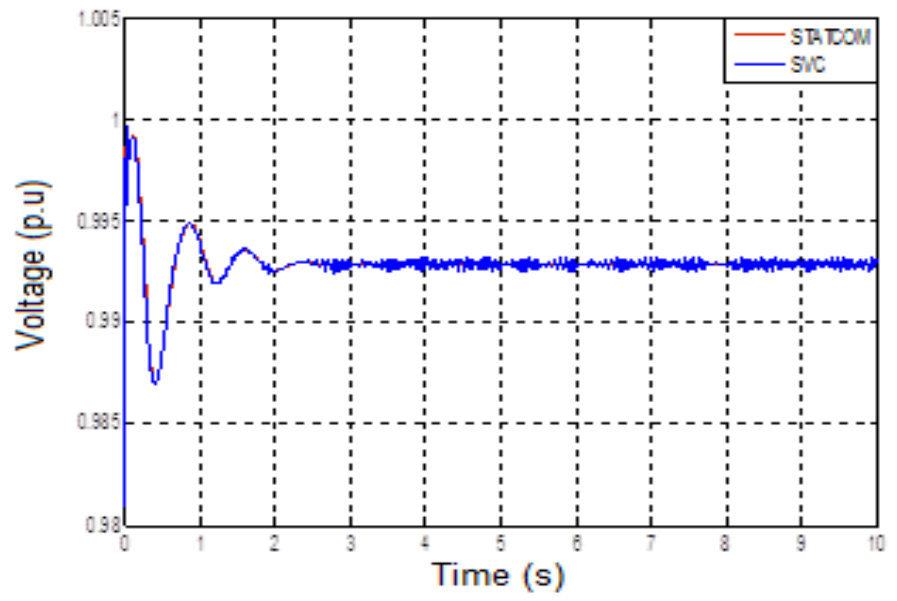

Figure 13: Voltage at PCC with FACTs

A double line to ground fault (DLG) followed by three phase to ground fault was simulated at PCC in this study. Improving the transient stability margin of the grid can be obtained by the utilization of the given two FACT devices (STATCOM, SVC). In order to compare the performance of these two FACT devices, three parameters were monitored during these two disturbances. These parameters are the voltage and reactive power at PCC and the induction generator rotor speed.
Voltage recovery time at $\mathrm{PCC}$ and the damping time period of rotor speed oscillation were compared relatively when FACTS devices (STATCOM, SVC) of equal converter rating are integrated to the grid. As seen from Fig. 14, the voltage before the occurrence of the fault was 0.954 p.u (pre-fault value) but after the DLG fault occurred, the voltage dipped down to zero at $\mathrm{t}=3 \mathrm{~s}$ then at $\mathrm{t}=3.2 \mathrm{~s}$ the voltage gets back to its pre-fault value. After 3 seconds which means at $t=6 s$, another 3 phase to ground fault has occurred at PCC. The fault recovery was at $\mathrm{t}=6.2 \mathrm{~s}$. It can be noted from the figure that even after the fault recovery, the voltage at PCC did not recover to its pre-fault value but stills approximately at 0.668 p.u.

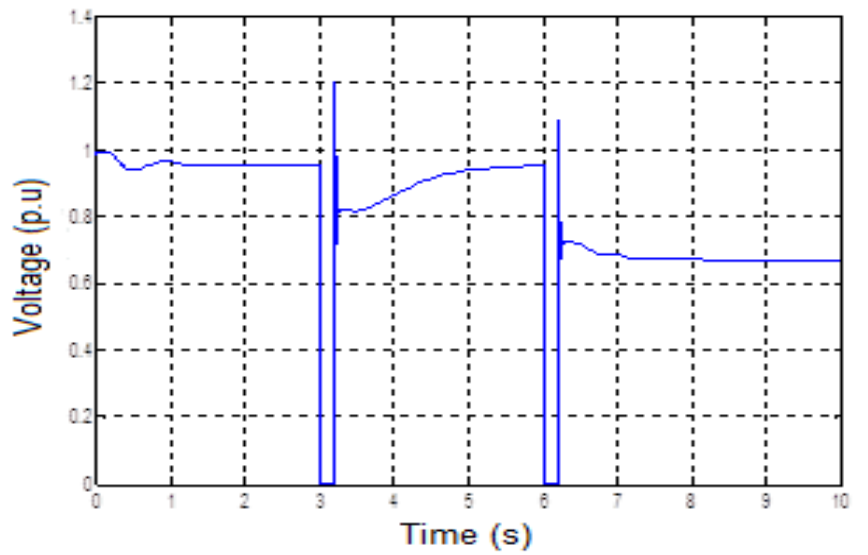

Figure 14. Voltage at PCC without FACTs

As a result of the voltage collapse or voltage instability which can be noticed from the last figure, the need for a dynamic var compensator is a must due to a large reactive power requirement by the wind generator during faulty conditions at PCC. A STATCOM and SVC can solve this problem. Fig. 15 depicts the voltage recovery performance of SVC and STATCOM due to faulty conditions at PCC.

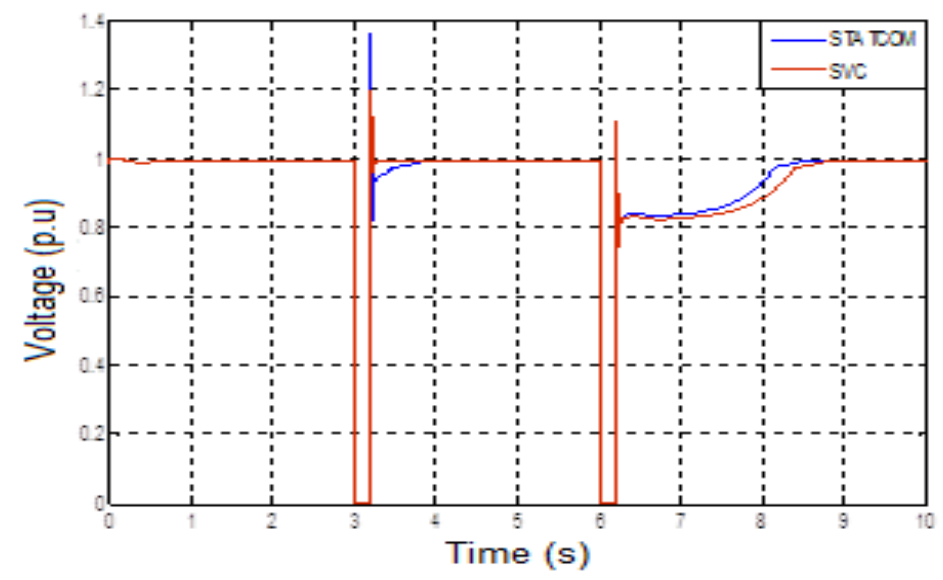

Figure 15: Voltage at PCC with FACTs

It is clearly shown that after the fault recovery, the voltage at PCC gets back to the pre-fault value. In maintaining voltage stability point of view or the voltage recovery time, It's also worth noting that the action of SVC is better than the action of STATCOM for the first disturbance (DLG) fault, while the 
action of STATCOM is better than SVC action for the second disturbance (3 phase- ground) fault.

The reactive power at PCC without the integration of FACTS devices can be seen in Fig. 16. It can be observed at PCC that the reactive power increases after the first disturbance to12 p.u and gets back to its pre-fault value after sometime. While for the second disturbance, it increases to approximately 16 p.u and never gets back to its pre-fault value.

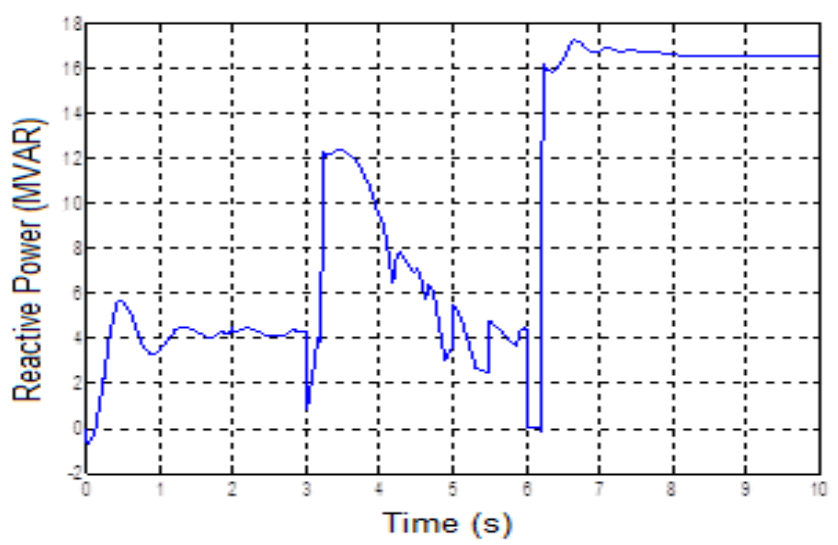

Figure 16: Reactive Power at PCC without FACTs

The reactive power at PCC with the integration of FACTS devices are shown in Fig. 17. It is clearly shown that the integration of FACTS devices reduce the reactive power for the two disturbances to approximately 1.5 MVAR which is the pre-fault value. It should also be noted that STATCOM is better and faster than SVC in recovering the reactive power to its pre-fault value for the second disturbance. But, SVC is better and faster than STATCOM in recovering the reactive power to its pre-fault value for the first disturbance.

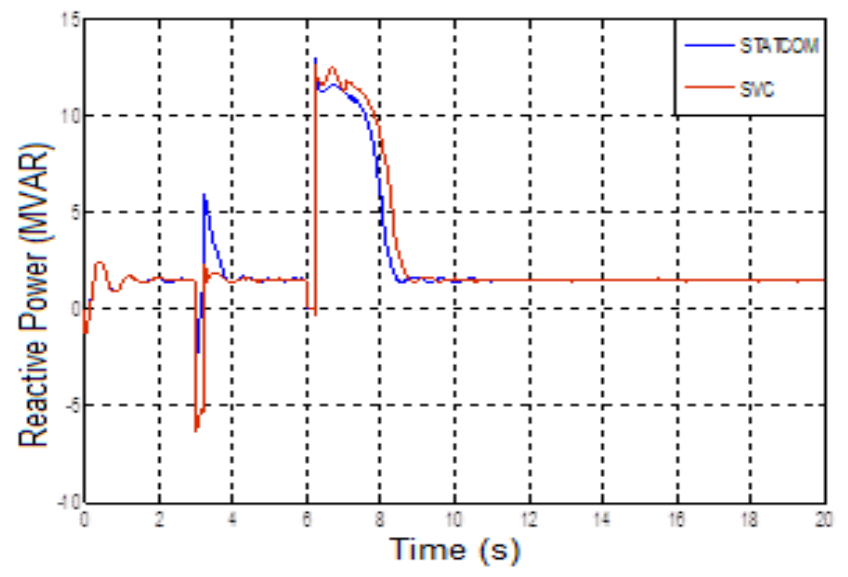

Figure 17: Reactive Power at PCC with FACTs

In addition to the reactive power and voltage at PCC, wind farm rotor speed is also monitored so as to determine its stability and its behavior with and without the integration of FACTS devices to the power system.

Fig. 18 shows the rotor speed of the wind generators without FACTS integration. It can be noticed from the figure that during the first disturbance, the rotor speed didn't change or increased. But, for the second disturbance the rotor speed increases rapidly and consequently the possibility of the generators to remain connected to the grid is almost impossible. This problem can be solved by the integration of FACTS devices at PCC as depicted in Fig. 19.

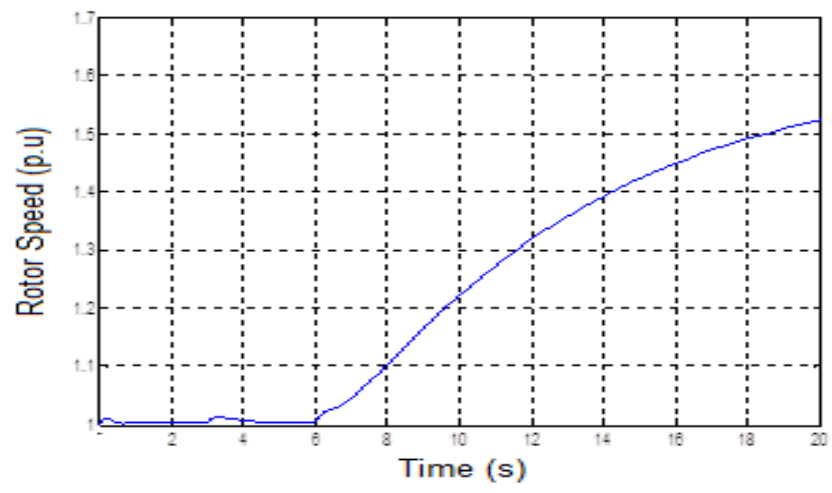

Figure 18: Wind generator rotor speed without FACTs

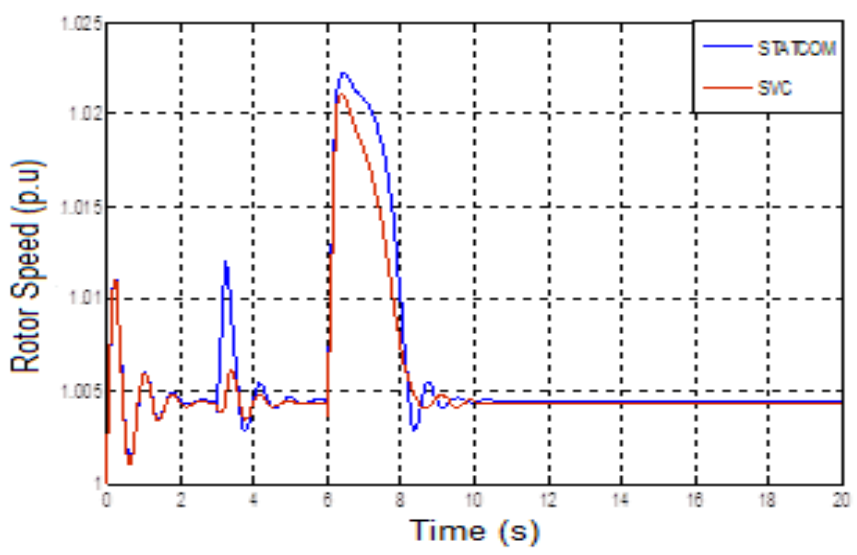

Figure 19: Wind generator rotor speed with FACTs

\section{CONCLUSION}

The performance of a wind farm equipped with shunt capacitor banks and both of the FACTS devices (STATCOM, SVC) which has equal converter ratings have been studied without taking into account the occurrence of any short circuits. Also at the same time, the occurrence of (DLG) fault followed by (3 phase to ground) fault at a point on the transmission line connecting the wind farm with the medium voltage grid has been studied. In healthy conditions (without short circuits), STATCOM and SVC have the same performance in improving the voltage stability of the system. However, in faulty conditions, STATCOM has better capability in voltage recovery and reactive power support than SVC for the second disturbance while it is the opposite for the first disturbance. Furthermore, both of them have approximately the same performance in stabilizing the wind farm generators rotor speed.

\section{REFERENCES}

[1] M.G.Sugirtha and Dr. P.Latha, "Analysis of Power Quality Problems in Grid connected wind Power Plant", International Conference on Recent Advancements in Electrical, Electronics and Control Engineering (ICONRAEeCE), pp. 19-24, Dec 2011.

[2] Dipesh. M. Patel, A. R. Nagera, and Dattesh Y. Joshi, "Power Quality Improvement with static compensator on grid integration of wind energy 
system”, International Conference on Engineering, Nirma University. (NUiCONE), 2011.

[3] F. Zhou, G. Joos, and C. Abhey, "voltage stability in weak connection wind farm", Power Engineering Society General Meeting, 2005. IEEE, vol. 2, pp. 1483- 1488, 12-16 June 2005.

[4] M.G.Sugirtha and Dr. P.Latha, "Analysis of Power Quality Problems in Grid connected wind Power Plant", International Conference on Recent Advancements in Electrical, Electronics and Control Engineering (ICONRAEeCE), pp. 19-24, Dec 2011.

[5] M. Tarafdar Hagh, A. Roshan Milani, A. Lafzi,"Dynamic Stability Improvement of a Wind Farm Connected to Grid Using STATCOM", 5th International Conference on Electrical Engineering/Electronics,Computer, Telecommunications and Information Technology, vol. 2, pp. 1057-1060, 2008.

[6] Mousa Marzband, Andreas Sumper, Mircea Chindris,"Modeling and Simulation of the Fixed Speed Wind Power Generation System for Grid Studies", 11th International Conference in Electrical Power Quality and Utilisation, pp. 1-6, 17-19, Oct 2011.

[7] Lie Xu, Liangzhong Yao, and Christian Sasse, "Comparison of using SVC and STATCOM for wind farm integration", International Conference on Power System Technology, pp. 1-7, Oct. 2006.

[8] Rajiv Singh, Asheesh Kumar Singh, and Ashutosh Kumar Singh. "Transient Stability Improvement of a FSIG Based Grid Connected wind Farm with the help of a SVC and a STATCOM: A Comparison", International Journal of Computer and Electrical Engineering, vol. 4, No. 1, pp. 55-59, Feb. 2012

[9] J.G. Slootweg, H. Polinder and W.L. Kling, "Representing wind turbine electrical generating systems in fundamental frequency simulations", IEEE Transactions on Energy Conversion, vol. 18, issue. 4, pp. 516524, Dec. 2003.

[10] Joselin Herbert, G.M.; Iniyan, S.; Sreevalsan, E.; Rajapandian, S. "A review of wind energy technologies", Renewable and Sustainable Energy Reviews, vol. 11, issue. 6, pp. 1117-1145, Aug. 2007.

[11] Sidhartha Panda, N.P.Padhy, "Investigating the impact of wind speed on active and reactive power penetration to the distribution network", International Journal of Electrical and Computer Engineering, Vol.3,No.13, pp. 224-229. Sep. 2008.

[12] N. G. Hingorani, L. Gyugyi, "Understanding FACTS; Concepts and Technology of Flexible AC Transmission Systems," IEEE Press book, 2000.

[13] M.J. Hossain, H.R. Pota, M. A. Mahmud and Rodrigo A. Ramos, "Impacts of Large-Scale Wind Generators Penetration on the Voltage Stability of Power Systems", Power and Energy Society General Meeting, IEEE, pp.1-8, 24-29 July 2011.

[14] Özgür Salih MUTLU, Lt., "Evaluating the Impacts of wind farms on power system operation", Journal of Naval Science and Engineering, vol. 6, No.2, pp. 166-185, Aug. 2010.

[15] S.A. Pastromas, "Grid support by wind turbines and future trends", Transmission and Distribution Conference and Exposition, IEEE PES, pp 1-7, 19-22 April 2010.

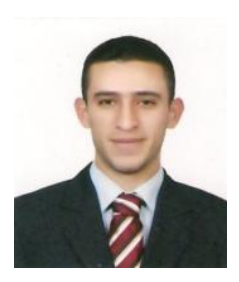

Qusay Salem was born in Irbid, Jordan, in 1986. He received the B.S degree in Electrical Power Engineering from Mutah University, the M.Sc degree in Electrical Power Engineering from Yarmouk University, Jordan in 2013. His research interests are in the areas of Stability of Power Systems connected to Distributed Generation, Smart Grid Applications, FACTS Devices Applications, Security of Power Systems, and Advanced Transmission Systems. 